\title{
Ranking Extensions in Abstract Argumentation
}

\author{
Kenneth Skiba $^{1}$, Tjitze Rienstra ${ }^{1}$, Matthias Thimm ${ }^{1}$, Jesse Heyninck ${ }^{2}$ and Gabriele \\ Kern-Isberner $^{2}$ \\ ${ }^{1}$ University of Koblenz-Landau, Koblenz, Germany \\ ${ }^{2}$ TU Dortmund, Dortmund, Germany \\ \{kennethskiba, rienstra, thimm\}@uni-koblenz.de, jesse.heyninck@tu-dortmund.de, \\ gabriele.kern-isberner@cs.tu-dortmund.de
}

\begin{abstract}
Extension-based semantics in abstract argumentation provide a criterion to determine whether a set of arguments is acceptable or not. In this paper, we present the notion of extension-ranking semantics, which determines a preordering over sets of arguments, where one set is deemed more plausible than another if it is somehow more acceptable. We obtain extension-based semantics as a special case of this new approach, but it also allows us to make more fine-grained distinctions, such as one set being "more complete" or "more admissible" than another. We define a number of general principles to classify extension-ranking semantics and develop concrete approaches. We also study the relation between extension-ranking semantics and argument-ranking semantics, which rank individual arguments instead of sets of arguments.
\end{abstract}

\section{Introduction}

Formal argumentation [Atkinson et al., 2017] is concerned with models of rational decision-making based on representations of arguments and their relationships. A particular important approach is that of abstract argumentation [Dung, 1995], which represents argumentative settings as directed graphs. Here, arguments are identified by vertices and an attack from one argument to another is represented as a directed edge. Reasoning is usually performed in abstract argumentation by considering extensions, i. e., sets of arguments that are jointly acceptable given some formal account of "acceptability". Therefore, this classical approach differentiates between "acceptable" arguments and "rejected" arguments.

A number of approaches have been developed aiming at determining the relative degree of acceptance of the arguments of an argumentation framework. In these approaches, a ranking-based, graded or gradual semantics associates every argumentation framework with an ordering of the arguments according to their relative degree of justification or acceptability [Amgoud and Ben-Naim, 2013; Amgoud et al., 2016; Bonzon et al., 2016; Yun et al., 2018; Grossi and Modgil, 2019]. In this paper, we contribute to this line of research but we take a different approach. An extension-ranking semantics $\tau$ maps an argumentation framework AF to a preorder $\preceq_{\mathrm{AF}}^{\tau}$ over all extensions (sets of arguments). Intuitively, for two extensions $E, E^{\prime}$ of AF, $E \preceq$ AF $E^{\prime}$ holds whenever $E$ is at least as plausible as $E^{\prime}$. While any extension-based seman$\operatorname{tics}^{1} \sigma$ can be defined in terms of an extension-ranking semantics -i. e. by defining the extension of AF to be the minimal elements according to $\preceq_{A F}^{\tau}$ - the general framework of extension-ranking semantics is more expressive than that. For instance, an extension-ranking semantics can be used to compare extensions that are not complete, admissible, or even conflict-free in the classical sense. Moreover, an extensionranking semantics retains the ability to represent constellations of arguments that can jointly be acceptable, which is not possible if we define the semantics of an argumentation framework in terms of a single ranking over arguments.

Extension-ranking semantics have a number of potential applications. One is decision making under constraints, where we need solutions (typically represented by extensions of an argumentation framework) that satisfy constraints that may not be satisfiable by the set of extensions under an extension semantics. In such a situation we can select the most plausible extensions among those that satisfy the constraint. Another application is belief dynamics [Booth et al., 2013; Coste-Marquis et al., 2014; Diller et al., 2018], where rankings over extensions can be used as a relation of epistemic entrenchment. Other problems where the ability to compare extensions is useful are belief merging and judgment aggregation [Delobelle et al., 2016; Caminada and Pigozzi, 2011]. In this work we take a first step towards this matter by (1) proposing a number of general principles for extension-ranking semantics, and (2) proposing a number of extension-ranking semantics that generalise admissible, complete, grounded, preferred and stable semantics. Finally, we show how an extension-ranking semantics can be used to define an argument ranking.

This paper is structured as follows. In Section 2 we recall the necessary basics of abstract argumentation theory. In Section 3 we introduce the notion of extension-ranking semantics. We then propose a number of general principles for extension-ranking semantics in Section 4 and a number of instances of extension-ranking semantics in Section 5. Section 6 deals with the connection between extension-ranking semantics and argument rankings. We discuss related work in Section 7 and conclude in Section 8.

\footnotetext{
${ }^{1}$ In the remainder of this paper, we refer to extension-based semantics simply as extension semantics to unify notation with extension-ranking semantics.
} 


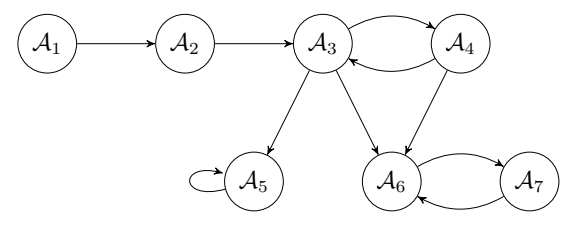

Figure 1: Abstract argumentation framework $A F_{1}$ from Example 1.

\section{Preliminaries}

An argumentation framework $\mathrm{AF}$ is a pair $\mathrm{AF}=(\mathrm{Arg}, R)$ where Arg is a set of arguments and $R$ is a relation $R \subseteq$ $\operatorname{Arg} \times \operatorname{Arg}[$ Dung, 1995]. We assume that Arg is finite. An argument $\mathcal{A}$ is said to attack an argument $\mathcal{B}$ if $(\mathcal{A}, \mathcal{B}) \in R$. We say that an argument $\mathcal{A}$ is defended by a set $E \subseteq \operatorname{Arg}$ if every argument $\mathcal{B} \in \operatorname{Arg}$ that attacks $\mathcal{A}$ is attacked by some $\mathcal{C} \in E$. For $\mathcal{A} \in \operatorname{Arg}$ define $\mathcal{A}^{-}=\{\mathcal{B} \mid(\mathcal{B}, \mathcal{A}) \in R\}$ and $\mathcal{A}^{+}=\{\mathcal{B} \mid(\mathcal{A}, \mathcal{B}) \in R\}$, so the sets of attackers of $\mathcal{A}$ and the set of arguments attacked by $\mathcal{A}$. For a set of arguments $E \subseteq \operatorname{Arg}$ we extend these sets by defining $E^{+}$and $E^{-}$via $E^{+}=\bigcup_{\mathcal{A} \in E} \mathcal{A}^{+}$and $E^{-}=\bigcup_{\mathcal{A} \in E} \mathcal{A}^{-}$, respectively.

An extension semantics determines a set of extensions (i. e., jointly acceptable sets of arguments) of an argumentation framework. Given an argumentation framework AF = $(\operatorname{Arg}, R)$, an extension $E \subseteq$ Arg is called admissible (ad) if and only if $E$ is conflict-free (cf), i. e., there are no arguments $\mathcal{A}, \mathcal{B} \in E$ with $(\mathcal{A}, \mathcal{B}) \in R$, and $E$ defends every $\mathcal{A} \in E$, and is called complete (co) if, additionally, it satisfies if $E$ defends $\mathcal{A}$ then $\mathcal{A} \in E$. A complete extension $E$ is grounded (gr) if and only if $E$ is $\subseteq$-minimal; is preferred (pr) if and only if $E$ is $\subseteq$-maximal; is stable (st) if and only if $\operatorname{Arg}=E \cup E^{+}$. Note that the grounded extension is uniquely determined and that stable extensions may not exist [Dung, 1995]. The grounded extension can also be defined as the least fixed point of the characteristic function. Given an argumentation framework $\mathrm{AF}=(\operatorname{Arg}, R)$, the characteristic function $F_{\mathrm{AF}}: 2^{\mathrm{Arg}} \rightarrow 2^{\mathrm{Arg}}$ is defined via

$$
F_{\mathrm{AF}}(E)=\{\mathcal{A} \in \operatorname{Arg} \mid E \text { defends } \mathcal{A}\}
$$

Given $\sigma \in\{\mathrm{cf}, \mathrm{ad}, \mathrm{co}, \mathrm{gr}, \mathrm{pr}, \mathrm{st}\}$ and argumentation framework AF we denote the set of all $\sigma$-extensions by $\sigma(\mathrm{AF}) .^{2}$

Example 1. Consider the abstract argumentation framework $A F_{1}$ depicted as a directed graph in Figure $1 . A F_{1}$ has four complete extensions $E_{1}=\left\{\mathcal{A}_{1}\right\}, E_{2}=\left\{\mathcal{A}_{1}, \mathcal{A}_{7}\right\}, E_{3}=$ $\left\{\mathcal{A}_{1}, \mathcal{A}_{3}, \mathcal{A}_{7}\right\}$, and $E_{4}=\left\{\mathcal{A}_{1}, \mathcal{A}_{4}, \mathcal{A}_{7}\right\} . E_{1}$ is the grounded extension, while $E_{3}$ and $E_{4}$ are both preferred, but only $E_{3}$ is stable.

\section{Extension-Ranking Semantics}

An extension semantics $\sigma$ provides a simple way to assess whether a given set of arguments is acceptable: either an extension $E$ is a $\sigma$-extension or not. In this section, we take a more general perspective on this issue by considering preorders over extensions.

Definition 1. An extension ranking on AF is a preorder ${ }^{3} \preceq$ over the powerset of arguments $2^{\mathrm{Arg}}$. An extension-ranking

\footnotetext{
${ }^{2}$ Although typically not considered a semantics, we treat of and ad as such, to simplify presentation.

${ }^{3} \mathrm{~A}$ preorder is a (binary) relation that is reflexive ( $E \preceq E$ for all $E$ ) and transitive ( $E_{1} \preceq E_{2}$ and $E_{2} \preceq E_{3}$ implies $\left.E_{1} \preceq E_{3}\right)$.
}

semantics $\tau$ is a function that maps each AF to an extension ranking $\preceq_{\mathrm{AF}}^{\tau}$ on $\mathrm{AF} .4$

Note that extension rankings are not necessarily total. For an extension-ranking semantics $\tau$, an extension ranking $\preceq_{\mathrm{AF}}^{\tau}$, $E, E^{\prime} \subseteq \mathrm{Arg}$, and for $E \preceq_{\mathrm{AF}}^{\tau} E^{\prime}$ we say that $E$ is at least as plausible as $E^{\prime}$ by $\tau$ in AF. We introduce the usual abbreviations: $E$ is strictly more plausible than $E^{\prime}$, denoted $E \prec_{\mathrm{AF}}^{\tau} E^{\prime}$, if $E \preceq_{\mathrm{AF}}^{\tau} E^{\prime}$ but not $E^{\prime} \preceq_{\mathrm{AF}}^{\tau} E$; $E$ and $E^{\prime}$ are incomparable, denoted $E \asymp_{\mathrm{AF}}^{\tau} E^{\prime}$, if neither $E \preceq_{\mathrm{AF}}^{\tau} E^{\prime}$ nor $E^{\prime} \preceq_{\mathrm{AF}}^{\tau} E$; and $E$ and $E^{\prime}$ are equally plausible, denoted $E \equiv_{\mathrm{AF}}^{\tau} E^{\prime}$, if $E \preceq_{\mathrm{AF}}^{\tau} E^{\prime}$ and $E^{\prime} \preceq_{\mathrm{AF}}^{\tau} E$.

Definition 2. We denote by $\min _{\tau}(\mathrm{AF}$ ) the minimal (or most plausible) elements of $\preceq_{\mathrm{AF}}^{\tau}$, i. e., $\min _{\tau}(\mathrm{AF})=\{E \subseteq$ $\operatorname{Arg} \mid \nexists E^{\prime} \subseteq$ Arg with $\left.E^{\prime} \prec_{\mathrm{AF}}^{\tau} E\right\}$.

Extension-ranking semantics provide an expressive semantical framework that generalises extension semantics. In fact, extension semantics can directly be used to define very naive instances of extension-ranking semantics as follows.

Definition 3. Given an extension semantics $\sigma$, we define the least-discriminating extension-ranking semantics wrt. $\sigma$, denoted $L D^{\sigma}$ by: $E \prec_{\mathrm{AF}}^{L D^{\sigma}} E^{\prime}$ if $E \in \sigma(\mathrm{AF})$ and $E^{\prime} \notin \sigma(\mathrm{AF})$; and $E \equiv_{\mathrm{AF}}^{L D^{\sigma}} E^{\prime}$, if $E, E^{\prime} \in \sigma(\mathrm{AF})$ or $E, E^{\prime} \notin \sigma(\mathrm{AF})$.

Our aim with extension-ranking semantics is to provide a finer distinction between extensions than extension semantics. In this paper, we focus on extension-ranking semantics that refine least-discriminating extension-ranking semantics wrt. $\sigma$ by providing a more fine-grained differentiation of those extensions that are not $\sigma$-extensions. In other words, for any two extensions that are no $\sigma$-extensions, we want to be able to say that one of them may be "closer" to being a $\sigma$ extension than the other. A complementary approach, which we deal with in future work, is to provide a more fine-grained differentiation of $\sigma$-extensions instead.

\section{Principles for Extension-Ranking Semantics}

In this section, we define a number of general principles to describe various aspects of an intuitively well-behaved extension-ranking semantics. The first set of principles aims at relating extension-ranking semantics with extension semantics. The $\sigma$-generalisation principle states that the most plausible extensions of an argumentation framework are exactly the $\sigma$-extensions of the argumentation framework. We also distinguish $\sigma$-soundness (i.e., the most plausible extensions are $\sigma$-extensions) and $\sigma$-completeness (i.e., every $\sigma$ extension is a most plausible extension).

Definition 4. Let $\sigma$ be an extension semantics and $\tau$ an extension-ranking semantics. $\tau$ satisfies

- $\sigma$-soundness iff for all AF: $\min _{\tau}(\mathrm{AF}) \subseteq \sigma(\mathrm{AF})$.

- $\sigma$-completeness iff for all AF: $\min _{\tau}(\mathrm{AF}) \supseteq \sigma(\mathrm{AF})$.

- $\sigma$-generalisation iff $\tau$ satisfies both $\sigma$-soundness and $\sigma$ completeness.

The least-discriminating extension-ranking semantics satisfy their corresponding generalisation principles:

\footnotetext{
${ }^{4}$ Our use of the term ranking does not imply that we work with total orders. This is consistent with terminology used in the literature on argument-ranking semantics (see, e.g., [Bonzon et al., 2016]).
} 
Proposition 1. $\preceq_{\mathrm{AF}}^{L D^{\sigma}}$ satisfies $\sigma$-generalisation.

We now consider additional principles pertaining to independence of different parts of an argumentation framework. Composition states that after combining two disjoint argumentation frameworks into one, the extension ranking follows the rankings on the restricted AFs. The other way round, decomposition postulates that after splitting an argumentation framework into two disjoint argumentation frameworks, the rankings on the restricted sub-AFs obey the ranking on the total AF.

Definition 5. Let $\tau$ be an extension-ranking semantics. ${ }^{5}$

- $\tau$ satisfies composition if for every AF such that $\mathrm{AF}=$ $\left(\operatorname{Arg}_{1}, R_{1}\right) \cup\left(\operatorname{Arg}_{2}, R_{2}\right)$ and $E, E^{\prime} \subseteq \operatorname{Arg}_{1} \cup \operatorname{Arg}_{2}$ : if $\left\{\begin{array}{ll}E \cap \operatorname{Arg}_{1} \preceq_{A F_{1}}^{\tau} & E^{\prime} \cap \operatorname{Arg}_{1} \\ E \cap \operatorname{Arg}_{2} \preceq_{\mathrm{AF}_{2}}^{\tau} & E^{\prime} \cap \operatorname{Arg}_{2}\end{array}\right\}$ then $E \preceq_{\mathrm{AF}}^{\tau} E^{\prime}$.

- $\tau$ satisfies decomposition if for every $\mathrm{AF}$ such that $\mathrm{AF}=$ $\left(\operatorname{Arg}_{1}, R_{1}\right) \cup\left(\operatorname{Arg}_{2}, R_{2}\right)$ and $E, E^{\prime} \subseteq \operatorname{Arg}_{1} \cup \operatorname{Arg}_{2}$ : if $E \preceq_{\mathrm{AF}}^{\tau} E^{\prime}$ then $\left\{\begin{array}{l}E \cap \operatorname{Arg}_{1} \preceq_{\mathrm{AF}_{1}}^{\tau} E^{\prime} \cap \operatorname{Arg}_{1} \\ E \cap \operatorname{Arg}_{2} \preceq_{\mathrm{AF}_{2}}^{\tau} E^{\prime} \cap \operatorname{Arg}_{2}\end{array}\right\}$.

An important property of rational accounts of argumentation is reinstatement, i. e., the ability to make an attacked argument acceptable by attacking its attackers. Complete semantics implements this property in a strict fashion: if an argument can be reinstated by an extension, it must be included in that extension. Therefore, an extension containing an argument it defends is more plausible than an extension without that argument. We define two different versions of this principle, a weak and a strong version. The weak version states that by adding a defended argument (that also introduces no further conflicts) we will not lower the plausibility of any extension. The strong version ensures, that by adding a reasonable argument to a set, the plausibility of that set will be strictly higher than before.

Definition 6. Let $\tau$ be an extension-ranking semantics.

- $\tau$ satisfies weak reinstatement iff $\mathcal{A} \in F_{\mathrm{AF}}(E), \mathcal{A} \notin E$ and $\mathcal{A} \notin\left(E^{-} \cup E^{+}\right)$implies $E \cup\{\mathcal{A}\} \preceq_{\mathrm{AF}}^{\tau} E$.

- $\tau$ satisfies strong reinstatement iff $\mathcal{A} \in F_{\mathrm{AF}}(E), \mathcal{A} \notin E$ and $\mathcal{A} \notin\left(E^{-} \cup E^{+}\right)$implies $E \cup\{\mathcal{A}\} \prec_{\mathrm{AF}}^{\tau} E$.

Note that the condition $\mathcal{A} \notin\left(E^{-} \cup E^{+}\right)$above is needed in order to not add additional conflicts to the extension (which may again lower the plausibility of the extension).

Finally, we define a principle similar in spirit to the $A b$ straction postulate from ranking-based semantics [Bonzon $e t$ $a l ., 2016]$. For that, an isomorphism $\gamma$ between two argumentation frameworks $\mathrm{AF}=(\operatorname{Arg}, R)$ and $\mathrm{AF}^{\prime}=\left(\operatorname{Arg}^{\prime}, R^{\prime}\right)$ is a bijective function $\gamma: \mathrm{AF} \rightarrow \mathrm{AF}^{\prime}$ such that $\left(\mathcal{A}_{1}, \mathcal{A}_{2}\right) \in R$ iff $\left(\gamma\left(\mathcal{A}_{1}\right), \gamma\left(\mathcal{A}_{2}\right)\right) \in R^{\prime}$ for all $\mathcal{A}_{1}, \mathcal{A}_{2} \in \operatorname{Arg}$. Our principle of syntax independence states that the names of each argument is not important for the resulting extension ranking.

Definition 7. An extension-ranking $\tau$ satisfies syntax independence if for every pair of $\mathrm{AFs} \mathrm{AF}=(\mathrm{Arg}, R), \mathrm{AF}^{\prime}=$ $\left(\mathrm{Arg}^{\prime}, R^{\prime}\right)$ and for every isomorphism $\gamma: \mathrm{AF} \rightarrow \mathrm{AF}^{\prime}$, for all $E, E^{\prime} \subseteq \operatorname{Arg}$, we have $E \preceq_{\mathrm{AF}}^{\tau} E^{\prime}$ iff $\gamma(E) \preceq_{\mathrm{AF}^{\prime}}^{\tau} \gamma\left(E^{\prime}\right)$.

${ }^{5}$ For $\mathrm{AF}_{1}=\left(\operatorname{Arg}_{1}, R_{1}\right)$ and $\mathrm{AF}_{2}=\left(\operatorname{Arg}_{2}, R_{2}\right)$ with $\operatorname{Arg}_{1} \cap$ $\mathrm{Arg}_{2}=\emptyset$. We define the disjoint union $\bullet$ as: $\mathrm{AF}_{1} \cup \mathrm{AF}_{2}=\left(\mathrm{Arg}_{1} \cup\right.$ $\left.\mathrm{Arg}_{2}, R_{1} \cup R_{2}\right)$

\section{Instances of Extension-Ranking Semantics}

In this section we propose a number of instances of extensionranking semantics. Each extension-ranking semantics generalises one of the main extension semantics, namely the admissible, complete, preferred, grounded, and (semi-)stable semantics.

\subsection{Base Functions}

To define the extension-ranking semantics, we first define four base functions that each focuses on modelling a single aspect of argumentative evaluation. These base functions and their corresponding base extension rankings will be combined later (in Sections 5.2-5.6) to obtain the desired generalisations. In Section 5.7 we evaluate these functions wrt. the principles discussed in the previous section.

The first aspect we consider is conflict-freeness, which is usually an undisputed and desirable property of every extension semantics. More concretely, a conflict-free extension is regarded as more plausible than a non-conflict-free extension. Generalising this idea, we deem an extension $E$ as more plausible than another extension $E^{\prime}$ if $E$ has strictly less conflicts than $E^{\prime}$ (wrt. set inclusion). We model this aspect with the following $C F$ base function and $C F$ base extension ranking (where $C F$ stands for "conflicts").

Definition 8. Let $\mathrm{AF}=(\operatorname{Arg}, R)$ and $E \subseteq \operatorname{Arg}$. Define the $C F$ base function $C F$ via

$$
C F(E, \mathrm{AF})=\{(\mathcal{A}, \mathcal{B}) \in R \mid \mathcal{A}, \mathcal{B} \in E\}
$$

and the corresponding $C F$ base extension ranking $\preceq_{\mathrm{AF}}^{C F}$ via

$$
E \preceq_{\mathrm{AF}}^{C F} E^{\prime} \text { iff } C F(E, \mathrm{AF}) \subseteq C F\left(E^{\prime}, \mathrm{AF}\right)
$$

Example 2. Consider again $A_{1}$ from Example 1. Let $E_{5}=\left\{\mathcal{A}_{2}, \mathcal{A}_{3}\right\}$ and $E_{6}=\left\{\mathcal{A}_{2}, \mathcal{A}_{3}, \mathcal{A}_{4}\right\}$. We have $C F\left(E_{5}, \mathrm{AF}_{1}\right)=\left\{\left(\mathcal{A}_{2}, \mathcal{A}_{3}\right)\right\}$ and $C F\left(E_{6}, \mathrm{AF}_{1}\right)=$ $\left\{\left(\mathcal{A}_{2}, \mathcal{A}_{3}\right),\left(\mathcal{A}_{3}, \mathcal{A}_{4}\right),\left(\mathcal{A}_{4}, \mathcal{A}_{3}\right)\right\}$. Hence $E_{5}$ is more plausible than $E_{6}$ with respect to their conflicts, $E_{5} \prec_{A F_{1}}^{C F} E_{6}$.

Another generally desirable property is admissibility. More precisely, we consider an extension defending all its elements as more plausible than an extension containing at least one undefended argument. Hence, an extension $E$ is more plausible than another extension $E^{\prime}$ if $E$ has strictly less undefended arguments than $E^{\prime}$ (wrt. set inclusions). The following $U D$ base function and $U D$ base extension ranking ( $U D$ stands for "undefended") capture this idea.

Definition 9. Let $\mathrm{AF}=(\operatorname{Arg}, R)$ and $E \subseteq \operatorname{Arg}$. Define the $U D$ base function $U D$ via

$$
U D(E, \mathrm{AF})=E \backslash F_{\mathrm{AF}}(E)
$$

and the corresponding $U D$ base extension ranking $\preceq_{A F}^{U D}$ via

$$
E \preceq_{\mathrm{AF}}^{U D} E^{\prime} \text { iff } U D(E, \mathrm{AF}) \subseteq U D\left(E^{\prime}, \mathrm{AF}\right)
$$

A complete extension contains every argument it defends. Hence, an extension containing all its defended arguments (without adding conflicts) is more plausible, than an extension not containing an argument that is actually defended. More generally speaking, an extension $E$ is more plausible than another extension $E^{\prime}$ if there are less arguments consistently defended by $E$ and not contained in $E$ than there are for 


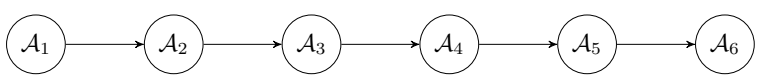

Figure 2: Abstract argumentation framework $A F_{2}$ from Example 3.

$E^{\prime}$ (wrt. set inclusion). By consistent defense we mean arguments that are defended by $E$ and do not attack $E$. In order to model this notion of consistent defense adequately, we need a more general notion of defense than what it is provided by the characteristic function $F_{\mathrm{AF}}$.

Example 3. Consider the argumentation framework $\mathrm{AF}_{2}$ depicted in Figure 2 and the extension $E_{1}=\left\{\mathcal{A}_{2}\right\}$. Observe that $F_{\mathrm{AF}_{2}}\left(E_{1}\right)=\left\{\mathcal{A}_{1}, \mathcal{A}_{4}\right\}$. However, given that we wish to determine the set of arguments that are consistently defended by $E_{1}$ we expect as outcome the set $\left\{\mathcal{A}_{2}, \mathcal{A}_{4}\right\}$ since $\mathcal{A}_{1}$ attacks a member of our initial set and should be ignored, therefore also leaving $\mathcal{A}_{2}$ within the outcome. Iterating the notion of consistent defense we end up with the set $\left\{\mathcal{A}_{2}, \mathcal{A}_{4}, \mathcal{A}_{6}\right\}$ modelling the intuition that $\mathcal{A}_{4}$ and $\mathcal{A}_{6}$ are consistently defended when we assume that $\mathcal{A}_{2}$ is accepted.

The function $F^{*}$ implements the above intuition as follows.

Definition 10. The function $F_{\mathrm{AF}}^{*}: 2^{\mathrm{Arg}} \rightarrow 2^{\mathrm{Arg}}$ is defined via

$$
F_{\mathrm{AF}}^{*}(E)=\bigcup_{i=1}^{\infty} F_{i, \mathrm{AF}}^{*}(E)
$$

with

$$
\begin{aligned}
F_{1, \mathrm{AF}}^{*}(E) & =E \\
F_{i, \mathrm{AF}}^{*}(E) & =F_{i-1, \mathrm{AF}}^{*}(E) \cup F_{\mathrm{AF}}\left(F_{i-1, \mathrm{AF}}^{*}(E)\right) \backslash E^{-}
\end{aligned}
$$

Applying $F^{*}$ on Example 3 we get as desired $F_{\mathrm{AF}_{2}}^{*}\left(\left\{\mathcal{A}_{2}\right\}\right)=\left\{\mathcal{A}_{2}, \mathcal{A}_{4}, \mathcal{A}_{6}\right\}$.

Finally we can define the $D N$ base function, that models the concept of consistent defense ( $D N$ meaning "consistent defended and not in").

Definition 11. Let $A F=(\operatorname{Arg}, R)$ and $E \subseteq \operatorname{Arg}$. We define the $D N$ base function $D N$ via

$$
D N(E, \mathrm{AF})=F^{*}(E)_{\mathrm{AF}} \backslash E
$$

and the corresponding $D N$ base extension ranking $\preceq_{\mathrm{AF}}^{D N}$ via

$$
E \preceq_{\mathrm{AF}}^{D N} E^{\prime} \text { iff } D N(E, \mathrm{AF}) \subseteq D N\left(E^{\prime}, \mathrm{AF}\right) .
$$

The final property we will take a look at is stability. A set is stable, if it attacks every argument not contained in it. To generalise this concept we state that an extension $E$ is more plausible than another extension $E^{\prime}$ if $E$ attacks strictly more arguments than $E^{\prime}$ (wrt. set inclusion). We capture this aspect with the $U A$ base function and the $U A$ base extension ranking ( $U A$ meaning "unattacked").

Definition 12. Let $A F=(\operatorname{Arg}, R)$ and $E \subseteq \operatorname{Arg}$. Define the $U A$ base function $U A$ via

$$
U A(E, \mathrm{AF})=\{\mathcal{A} \in \operatorname{Arg} \backslash E \mid \neg \exists \mathcal{B} \in E:(\mathcal{B}, \mathcal{A}) \in R\}
$$

and the corresponding $U A$ base extension ranking $\preceq_{\mathrm{AF}}^{U A}$ via

$$
E \preceq_{\mathrm{AF}}^{U A} E^{\prime} \text { iff } U A(E, \mathrm{AF}) \subseteq U A\left(E^{\prime}, \mathrm{AF}\right)
$$

\subsection{Admissible Extension-Ranking Semantics}

By combining the base extension-rankings defined in the previous section, we can define different extension-ranking semantics that generalise the extension semantics defined in Section 2. We start with the admissible extension-ranking semantics. Recall that an admissible extension is conflict-free and defends all its elements. We generalise this by assessing those extensions as more plausible that feature fewer conflicts and fewer undefended arguments. This is achieved by the lexicographic combination of the $\preceq_{\mathrm{AF}}^{C F}$ and $\preceq_{\mathrm{AF}}^{U D}$-rankings.

Definition 13. Let $A F=(\operatorname{Arg}, R)$ and $E, E^{\prime} \subseteq$ Arg. Define the admissible extension-ranking semantics $\mathrm{r}$-ad via

$$
E \preceq_{\mathrm{A} F}^{\mathrm{r} \text {-ad }} E^{\prime} \text { iff } E \prec_{\mathrm{AF}}^{C F} E^{\prime} \text { or }\left(E \equiv_{\mathrm{AF}}^{C F} E^{\prime} \text { and } E \preceq_{\mathrm{AF}}^{U D} E^{\prime}\right)
$$

Example 4. Consider $A F_{1}$ from Example 1. The sets $E_{7}=\left\{\mathcal{A}_{2}\right\}$ and $E_{8}=\left\{\mathcal{A}_{2}, \mathcal{A}_{6}\right\}$ are both conflict-free, but not admissible. However $U D\left(E_{7}, \mathrm{AF}_{1}\right)=\left\{\mathcal{A}_{2}\right\}$ and $U D\left(E_{8}, \mathrm{AF}_{1}\right)=\left\{\mathcal{A}_{2}, \mathcal{A}_{6}\right\}$, so $E_{7} \preceq_{\mathrm{AF}_{1}}^{\mathrm{r}-\mathrm{ad}} E_{8}$.

Since the most conflicting extension $E_{\text {all }}=\operatorname{Arg}$ is a most plausible extension for every base function except $\preceq_{A F}^{C F}$ we use the lexicographic combination of first the $\preceq_{\mathrm{AF}}^{C F}$-ranking and then the $\preceq_{\mathrm{AF}}^{U D}$-ranking to ensure that $E_{\text {all }}$ is always ranked worse, than any other extension $E^{\prime} \subset E_{\text {all }}$.

\subsection{Complete Extension-Ranking Semantics}

Recall that a complete extension is an admissible extension that includes all the arguments it defends. We generalise this by favouring extensions that are more admissible and, in case of equal admissibility, favouring extensions that include larger sets of defended arguments. This is achieved by the lexicographic combination of $\preceq_{\mathrm{AF}}^{\mathrm{r} \text {-ad }}$ and $\preceq_{\mathrm{AF}}^{D N}$-rankings.

Definition 14. Let $\mathrm{AF}=(\operatorname{Arg}, R)$ and $E, E^{\prime} \subseteq \mathrm{Arg}$. We define the complete extension-ranking semantics $r$-co via

$$
E \preceq \preceq_{\mathrm{AF}}^{\mathrm{r}-\mathrm{co}} E^{\prime} \text { iff } E \prec_{\mathrm{AF}}^{\mathrm{r} \text {-ad }} E^{\prime} \text { or }\left(E \equiv_{\mathrm{AF}}^{\mathrm{r} \text {-ad }} E^{\prime} \text { and } E \preceq_{\mathrm{AF}}^{D N} E^{\prime}\right)
$$

Example 5. Consider $\mathrm{AF}_{1}$ from Example 1. The sets $E_{9}=$ $\left\{\mathcal{A}_{7}\right\}$ and $E_{10}=\left\{\mathcal{A}_{4}\right\}$ are both admissible, but not complete. However $D N\left(E_{9}, \mathrm{AF}_{1}\right)=\left\{\mathcal{A}_{1}\right\}$ and $U D\left(E_{10}, \mathrm{AF}_{1}\right)=$ $\left\{\mathcal{A}_{1}, \mathcal{A}_{7}\right\}$, so $E_{9} \preceq_{\mathrm{AF}_{1}}^{\mathrm{r} \text {-co }} E_{10}$.

\subsection{Grounded Extension-Ranking Semantics}

A grounded extension is a complete extension that is minimal with respect to set inclusion. We generalise this by favouring extensions that are more complete and, in case of equal completeness, favouring smaller extensions. This is achieved by the lexicographic combination of $\preceq_{\mathrm{AF}}^{\mathrm{r} \text {-co }}$ - and $\subseteq$-rankings.

Definition 15. Let $\mathrm{AF}=(\operatorname{Arg}, R)$ and $E, E^{\prime} \subseteq \mathrm{Arg}$. We define the grounded extension-ranking semantics $r$-gr via

$$
E \preceq_{\mathrm{AF}}^{\mathrm{r}-\mathrm{gr}} E^{\prime} \text { iff } E \prec_{\mathrm{AF}}^{\mathrm{r}-\mathrm{co}} E^{\prime} \text { or }\left(E \equiv_{\mathrm{AF}}^{\mathrm{r}-\mathrm{co}} E^{\prime} \text { and } E \subseteq E^{\prime}\right)
$$

Example 6. Consider $\mathrm{AF}_{1}$ from Example 1. The sets $E_{2}=$ $\left\{\mathcal{A}_{1}, \mathcal{A}_{7}\right\}$ and $E_{3}=\left\{\mathcal{A}_{1}, \mathcal{A}_{3}, \mathcal{A}_{7}\right\}$ are both complete, but not grounded. However $E_{2} \subset E_{3}$, so $E_{2} \preceq_{\mathrm{AF}_{1}}^{\mathrm{r}-\mathrm{gr}} E_{3}$. 


\subsection{Preferred Extension-Ranking Semantics}

Recall that a preferred extension is an admissible extension that is maximal with respect to set inclusion. Accordingly, the preferred extension-ranking semantics is based on favouring extensions that are more admissible and, in case of equal admissibility, favouring extensions that are larger with respect to set inclusion. This is achieved by the lexicographic combination of the $\preceq_{A F}^{r-a d}$ and $\supseteq$-rankings.

Definition 16. Let $\mathrm{AF}=(\mathrm{Arg}, R)$ and $E, E^{\prime} \subseteq \mathrm{Arg}$. We define the preferred extension-ranking semantics $\mathrm{r}$-pr via

$$
E \preceq_{\mathrm{AF}}^{\mathrm{r} \text {-pr }} E^{\prime} \text { iff } E \prec_{\mathrm{AF}}^{\mathrm{r} \text {-ad }} E^{\prime} \text { or }\left(E \equiv_{\mathrm{AF}}^{\mathrm{r} \text {-ad }} E^{\prime} \text { and } E^{\prime} \subseteq E\right)
$$

Example 7. We continue Example 1. There, the sets $E_{1}=$ $\left\{\mathcal{A}_{1}\right\}$ and $E_{2}=\left\{\mathcal{A}_{1}, \mathcal{A}_{7}\right\}$ are both complete, but not preferred. However $E_{2} \subset E_{1}$, so $E_{1} \preceq_{\mathrm{AF}_{1}}^{\mathrm{r}-\mathrm{pr}} E_{2}$.

\section{6 (Semi-)Stable Extension-Ranking Semantics}

Recall that a stable extension is a conflict-free extension that attacks all arguments not included in the extension. We can define a corresponding extension-ranking semantics that favours extensions that are more conflict-free and, in case of equal conflict-freeness, favours extensions that are minimal with respect to the set of arguments not included in the extension and not attacked by the extension. This is achieved by the lexicographic combination of the $\preceq_{A F}^{r-c o}$ and $\preceq_{A F}^{U A}$ rankings. However, the result is not st-sound, since not every argumentation framework has a stable extension. In fact, this extension-ranking semantics generalises the semi-stable semantics [Caminada et al., 2012].

Definition 17. For $\mathrm{AF}=(\operatorname{Arg}, R)$, an extension $E \subseteq \operatorname{Arg}$ is a semi-stable extension if and only if $E$ is a complete extension where $E \cup E^{+}$is maximal wrt. set inclusion.

Definition 18. Let $\mathrm{AF}=(\operatorname{Arg}, R)$ and $E, E^{\prime} \subseteq$ Arg. We define the semi-stable extension-ranking semantics $r$-sst via

$$
E \preceq_{\mathrm{AF}}^{\mathrm{r} \text {-sst }} E^{\prime} \text { iff } E \prec_{\mathrm{AF}}^{\mathrm{r} \text {-co }} E^{\prime} \text { or }\left(E \equiv_{\mathrm{AF}}^{\mathrm{r} \text { co }} E^{\prime} \text { and } E \preceq_{\mathrm{AF}}^{U A} E^{\prime}\right)
$$

Example 8. Consider $\mathrm{AF}_{1}$ from Example 1. The sets $E_{2}=$ $\left\{\mathcal{A}_{1}, \mathcal{A}_{7}\right\}$ and $E_{4}=\left\{\mathcal{A}_{1}, \mathcal{A}_{4}, \mathcal{A}_{7}\right\}$ are both complete, but not stable. However $U A\left(E_{2}, \mathrm{AF}_{1}\right)=\left\{\mathcal{A}_{3}, \mathcal{A}_{4}, \mathcal{A}_{5}\right\}$ and $U A\left(E_{4}, \mathrm{AF}_{1}\right)=\left\{\mathcal{A}_{5}\right\}$, so $E_{4} \underset{\mathrm{AF}_{1}}{\mathrm{r} \text {-sst }} E_{2}$.

\subsection{Compliance to Principles}

The following result summarises the compliance of our approaches with the principles defined in Section 4.

Theorem 1. An extension-ranking semantics $\tau \in$ $\{r$-ad, r-co, r-gr, r-pr, r-sst $\}$ satisfies the respective principles as stated in Table $1 .^{6}$

We see that every extension-ranking semantics satisfies only $\sigma$-generalisation for the extension semantics they are based on. Hence these extension-ranking semantics do indeed generalise extension-based reasoning.

Another important result is that every extension-ranking semantics satisfies composition and decomposition. This results in the desirable property that combining two independent argumentation frameworks will not change the plausibility of any extension.

\footnotetext{
${ }^{6}$ The proof of Theorem 1 can be found in an online appendix at http://mthimm.de/misc/proofs_ijcai21_srthk.pdf
}

\begin{tabular}{|c||c|c|c|c|c|}
\hline Principles & r-ad & r-co & r-gr & r-pr & r-sst \\
\hline \hline cf-generalisation & X & X & X & X & X \\
ad-generalisation & $\checkmark$ & X & X & X & X \\
co-generalisation & X & $\checkmark$ & X & X & X \\
gr-generalisation & X & X & $\checkmark$ & X & X \\
pr-generalisation & X & X & X & $\checkmark$ & X \\
sst-generalisation & X & X & X & X & $\checkmark$ \\
\hline composition & $\checkmark$ & $\checkmark$ & $\checkmark$ & $\checkmark$ & $\checkmark$ \\
decomposition & $\checkmark$ & $\checkmark$ & $\checkmark$ & $\checkmark$ & $\checkmark$ \\
\hline weak reinstatement & $\checkmark$ & $\checkmark$ & $\checkmark$ & $\checkmark$ & $\checkmark$ \\
strong reinstatement & X & $\checkmark$ & $\checkmark$ & $\checkmark$ & $\checkmark$ \\
\hline syntax independence & $\checkmark$ & $\checkmark$ & $\checkmark$ & $\checkmark$ & $\checkmark$ \\
\hline
\end{tabular}

Table 1: Extension-ranking semantics analysed with respect to principles. $\mathrm{X}$ means that a extension-ranking semantics does not satisfy a principle and $\checkmark$ means it does.

We see that all extension-ranking semantics defined above do satisfy the weak version of the reinstatement principle. Hence we can be sure that by adding a reasonable argument (i. e., an argument defended by the set such that its addition will not create new conflicts) we do not lower the plausibility of a set. Only the admissible extension-ranking semantics does not satisfy strong reinstatement. So strong reinstatement behaves similar as the reinstatement principle for extension semantics, as there also only the admissible semantics does not satisfy reinstatement (as extensions may be admissible that do not include all defended arguments).

Example 9. Consider the argumentation framework $\mathrm{AF}_{3}=$ $\left\{\left\{\mathcal{A}_{1}, \mathcal{A}_{2}, \mathcal{A}_{3}\right\},\left\{\left(\mathcal{A}_{1}, \mathcal{A}_{2}\right),\left(\mathcal{A}_{2}, \mathcal{A}_{3}\right\}\right\}\right.$. The sets $E_{1}=\left\{\mathcal{A}_{1}\right\}$ and $E_{2}=\left\{\mathcal{A}_{1}, \mathcal{A}_{3}\right\}$ are both admissible. By applying the admissible extension-ranking semantics to both sets, we get $E_{1} \equiv_{\mathrm{AF}_{3}}^{\mathrm{r} \text {-ad }} E_{2}$. However $\mathcal{A}_{3}$ is the only suitable candidate to be reinstated for $E_{1}$. But $E_{1} \cup\left\{\mathcal{A}_{3}\right\} \nprec_{A F_{3}}^{\text {r-ad }} E_{1}$, as they are equally plausible. So the admissible extension-ranking semantics does not satisfy strong reinstatement.

As every extension-ranking semantics defined as above never considers the names of the arguments, we see that all of them do satisfy syntax independence.

\section{Projection on Argument Ranking}

The approach of extension-ranking semantics can be also be used to define a ranking over arguments. The area of argument-ranking semantics has been researched over the last few years, see [Bonzon et al., 2016] for an overview. Here arguments are ordered based on their acceptability (i.e., argument $\mathcal{A}_{1}$ is more acceptable than $\mathcal{A}_{2}$ in an argumentation framework $\mathrm{AF}, \mathcal{A}_{1} \unlhd_{\mathrm{AF}} \mathcal{A}_{2}$ ). We project an extension ranking to an argument ranking as follows:

Definition 19. Let $\tau$ be an extension-ranking semantics and $\mathrm{AF}=(\mathrm{Arg}, R)$. We define an argument ranking $\unlhd_{\mathrm{AF}}^{\tau}$ via $\mathcal{A}_{1} \unlhd_{\mathrm{AF}}^{\tau} \mathcal{A}_{2}$ iff there is a set $E$ with $\mathcal{A}_{1} \in E$ s.t for all $E^{\prime}$ with $\mathcal{A}_{2} \in E^{\prime}$ we have $E \preceq_{\mathrm{AF}}^{\tau} E^{\prime}$.

In other words argument $\mathcal{A}_{1}$ is at least as acceptable as $\mathcal{A}_{2}$, if there is a set containing $\mathcal{A}_{1}$, which is at least as plausible as every set containing $\mathcal{A}_{2}$, with respect to an extension-ranking semantics $\tau$. 
Similar to the principles defined above, there exists a number of rationality postulates for argument-ranking semantics. Here, we will only recall three very simple ones, namely, $A b$ straction, Independence and Self-contradiction.

The postulate abstraction denotes, that names of arguments are not relevant for an argument-ranking.

Abstraction For every pair of $\mathrm{AFs} \mathrm{AF}=(\mathrm{Arg}, R), \mathrm{AF}^{\prime}=$ $\left(\mathrm{Arg}^{\prime}, R^{\prime}\right)$ and for every isomorphism $\gamma: \mathrm{AF} \rightarrow \mathrm{AF}^{\prime}$, for all $\mathcal{A}_{1}, \mathcal{A}_{2} \in \operatorname{Arg}$, we have $\mathcal{A}_{1} \unlhd_{\mathrm{AF}} \mathcal{A}_{2}$ iff $\gamma\left(\mathcal{A}_{1}\right) \unlhd_{\mathrm{AF}^{\prime}}$ $\gamma\left(\mathcal{A}_{2}\right)$.

The connected components of AF are the set of maximal subgraphs $A F^{\prime}$ of $A F$, denoted by $c c(A F)$, such that for every pair $\mathcal{A}_{1}, \mathcal{A}_{2} \in \mathrm{AF}^{\prime}$ there is a path connecting them (while ignoring the direction of the edges). With Independence we state, that every connected component behaves independently from each other wrt. their rankings.

Independence For every $\mathrm{AF}$ such that $\mathrm{AF}^{\prime} \in c c(\mathrm{AF})$, $\mathcal{A}_{1}, \mathcal{A}_{2} \in \mathrm{AF}^{\prime}, \mathcal{A}_{1} \unlhd_{\mathrm{AF}^{\prime}} \mathcal{A}_{2}$ iff $\mathcal{A}_{1} \unlhd_{\mathrm{AF}} \mathcal{A}_{2}$.

Self-contradiction denotes that self-attacking arguments shall be ranked lower than any other argument.

Self-contradiction For every argumentation framework $\mathrm{AF}=(\mathrm{Arg}, R)$ and for all $\mathcal{A}_{1}, \mathcal{A}_{2} \in \mathrm{Arg}$ with $\left(\mathcal{A}_{1}, \mathcal{A}_{1}\right) \notin R$ and $\left(\mathcal{A}_{2}, \mathcal{A}_{2}\right) \in R$ then $\mathcal{A}_{1} \preceq$ AF $\mathcal{A}_{2}$ and $\mathcal{A}_{2} \npreceq \mathrm{AF} \mathcal{A}_{1}$.

For any argument ranking defined by the transformation from Definition 19, using an extension-ranking semantics, which satisfies syntax independence, will satisfy abstraction since the names of the arguments are not important.

There is a connection between decomposition and independence, as both behave similar. This is because the restriction of a most plausible extension of an argumentation framework to a subgraph is also most plausible for that subgraph. Hence, an argument ranking constructed with the projection from Definition 19 satisfies independence, if the underlying extension-ranking semantics satisfies decomposition.

An argument ranking defined with the projection from Definition 19 satisfies Self-contradiction if the underlying extension-ranking semantics satisfies $c f$-soundness. This shows, that conflict-free extensions are always ranked higher, than not conflict-free sets. This implies that $\left\{\mathcal{A}_{1}\right\} \prec_{\mathrm{AF}}^{C F^{\prime}} E^{\prime}$, if $E^{\prime}$ contains a self-attacking argument.

Proposition 2. Let $\tau \in\{$ r-ad, r-co, r-gr, r-pr, r-sst $\}$ and $\mathrm{AF}=(\mathrm{Arg}, R)$. The argument ranking $\unlhd_{\mathrm{AF}}^{\tau}$ satisfies abstraction, independence and self-contradiction.

\section{Related Work}

A number of works study methods to order arguments according to their (relative) degree of acceptability [Amgoud et al., 2016; Amgoud and Ben-Naim, 2013; Bonzon et al., 2016; Grossi and Modgil, 2019]. In [Yun et al., 2018], a framework is developed to determine a ranking over a set of extensions, on the basis of a ranking over arguments. In the previous section we dealt with the converse problem, i.e., determining a ranking over arguments based on a ranking over extensions.

Orderings over labellings of argumentation frameworks have been considered in [Arieli and Rienstra, 2014], [Rienstra, 2014] and [Rienstra and Thimm, 2018]. The latter two works consider some general principles for such orderings, namely conditional directionality and SCC stratification, which are counterparts of the directionality and SCC decomposability principles used in abstract argumentation [Baroni et al., 2011]. In future work we plan to study similar principles in the current extension-based setting as well.

The works [Bonzon et al., 2018; Konieczny et al., 2015] also consider approaches to rank sets of arguments, but their focus is on providing a more fine-grained differentiation of sets that are extensions wrt. some semantics. In particular, [Konieczny et al., 2015] use such rankings to select the best extensions out of the set of all extensions. They do this by, e. g., promoting those extensions where the members are also ranked high by some given argument ranking. Similarly, [Bonzon et al., 2018] combines extension semantics with argument rankings in order to improve the argument ranking by the information provided by the extensions, and vice versa. So these approaches are somewhat complementary to our approach, since we focus on the problem of differentiating sets of arguments that are not extensions wrt. some semantics, cf. the property of $\sigma$-generalisation.

Several approaches to model belief change involve preorders over extensions or labellings of an argumentation framework [Booth et al., 2013; Coste-Marquis et al., 2014; Diller et al., 2018]. Roughly speaking, given an argumentation framework AF and extension semantics $\sigma$, a pre-order $\preceq$ over sets of arguments of AF is used where the minimal sets are the $\sigma$-extensions of AF, and the result of revision is determined by the minimal sets of arguments that satisfy the new information. In future work we plan to study the extension-ranking semantics that we defined in this context as well, by studying relationships between principles satisfied by the extension-ranking semantics, and postulates satisfied by corresponding revision operators.

\section{Conclusion}

In this work we presented an approach to generalise the extension-based reasoning process in abstract argumentation. This approach allows us to make a more fine-grained distinction between sets, based on their "completeness" or "admissiblity". We also developed concrete approaches as well as a number of general principles to classify these approaches. In the end we bridge the gap between extension-ranking semantics and argument-ranking semantics, by describing relationships between these two concepts.

As for future work we plan to define more principles and also develop a number of extension-ranking approaches, which are not based on the admissible semantics, like stage semantics [Verheij, 1996]. Further we can combine the rankings not only by lexicographic combination rather use different preference aggregation methods. Another possibility is to not only differentiate non- $\sigma$-extension, but also $\sigma$-extensions. Also we want to tighten the bond between extension-ranking and argument-ranking, as these two concepts are closely related.

\section{Acknowledgments}

The work reported here has been supported by the Deutsche Forschungsgemeinschaft (project numbers 375588274 and 423456621). 


\section{References}

[Amgoud and Ben-Naim, 2013] L. Amgoud and J. BenNaim. Ranking-based semantics for argumentation frameworks. In Proceedings of the 7th International Conference on Scalable Uncertainty Management (SUM'13), pages 134-147, 2013.

[Amgoud et al., 2016] Leila Amgoud, Jonathan Ben-Naim, Dragan Doder, and Srdjan Vesic. Ranking arguments with compensation-based semantics. In Principles of Knowledge Representation and Reasoning: Proceedings of the Fifteenth International Conference, KR 2016, Cape Town, South Africa, April 25-29, 2016, pages 12-21, 2016.

[Arieli and Rienstra, 2014] Ofer Arieli and Tjitze Rienstra. Preferential reasoning based on abstract argumentation semantics. In Computational Models of Argument - Proceedings of COMMA 2014, Atholl Palace Hotel, Scottish Highlands, UK, September 9-12, 2014, pages 77-88, 2014.

[Atkinson et al., 2017] K. Atkinson, P. Baroni, M. Giacomin, A. Hunter, H. Prakken, C. Reed, G.R. Simari, M. Thimm, and S. Villata. Toward artificial argumentation. AI Magazine, 38(3):25-36, October 2017.

[Baroni et al., 2011] Pietro Baroni, Martin Caminada, and Massimiliano Giacomin. An introduction to argumentation semantics. Knowledge Engineering Review, 26(4):365-410, 2011.

[Bonzon et al., 2016] Elise Bonzon, Jérôme Delobelle, Sébastien Konieczny, and Nicolas Maudet. A comparative study of ranking-based semantics for abstract argumentation. In Thirtieth AAAI Conference on Artificial Intelligence, 2016.

[Bonzon et al., 2018] Elise Bonzon, Jérôme Delobelle, Sébastien Konieczny, and Nicolas Maudet. Combining extension-based semantics and ranking-based semantics for abstract argumentation. In 16th International Conference on Principles of Knowledge Representation and Reasoning, 2018.

[Booth et al., 2013] Richard Booth, Souhila Kaci, Tjitze Rienstra, and Leendert W. N. van der Torre. A logical theory about dynamics in abstract argumentation. In Scalable Uncertainty Management - 7th International Conference, SUM 2013, Washington, DC, USA, September 16-18, 2013. Proceedings, pages 148-161, 2013.

[Caminada and Pigozzi, 2011] Martin Caminada and Gabriella Pigozzi. On judgment aggregation in abstract argumentation. Auton. Agents Multi Agent Syst., 22(1):64-102, 2011.

[Caminada et al., 2012] Martin W. A. Caminada, Walter Alexandre Carnielli, and Paul E. Dunne. Semi-stable semantics. J. Log. Comput., 22(5):1207-1254, 2012.

[Coste-Marquis et al., 2014] Sylvie Coste-Marquis, Sébastien Konieczny, Jean-Guy Mailly, and Pierre Marquis. On the revision of argumentation systems: Minimal change of arguments statuses. In Proceedings of the 14th International Conference on Principles of Knowledge Representation and Reasoning (KR'14), 2014.

[Delobelle et al., 2016] Jérôme Delobelle, Adrian Haret, Sébastien Konieczny, Jean-Guy Mailly, Julien Rossit, and
Stefan Woltran. Merging of abstract argumentation frameworks. KR, 2016(33-42):7, 2016.

[Diller et al., 2018] Martin Diller, Adrian Haret, Thomas Linsbichler, Stefan Rümmele, and Stefan Woltran. An extension-based approach to belief revision in abstract argumentation. Int. J. Approx. Reason., 93:395-423, 2018.

[Dung, 1995] Phan Minh Dung. On the Acceptability of Arguments and its Fundamental Role in Nonmonotonic Reasoning, Logic Programming and n-Person Games. Artificial Intelligence, 77(2):321-358, 1995.

[Grossi and Modgil, 2019] Davide Grossi and Sanjay Modgil. On the graded acceptability of arguments in abstract and instantiated argumentation. Artificial Intelligence, 275:138-173, October 2019.

[Konieczny et al., 2015] Sébastien Konieczny, Pierre Marquis, and Srdjan Vesic. On supported inference and extension selection in abstract argumentation frameworks. In European Conference on Symbolic and Quantitative Approaches to Reasoning and Uncertainty, pages 49-59. Springer, 2015.

[Rienstra and Thimm, 2018] Tjitze Rienstra and Matthias Thimm. Ranking functions over labellings. In Sanjay Modgil, Katarzyna Budzynska, and John Lawrence, editors, Proceedings of the Seventh International Conference on Computational Models of Argumentation (COMMA'18), volume 305 of Frontiers in Artificial Intelligence and Applications, pages 393-404, Warsaw, Poland, September 2018.

[Rienstra, 2014] Tjitze Rienstra. Argumentation in Flux Modelling Change in the Theory of Argumentation. $\mathrm{PhD}$ thesis, University of Luxembourg/University of Montpellier II, 2014.

[Verheij, 1996] Bart Verheij. Two approaches to dialectical argumentation: admissible sets and argumentation stages. Proc. NAIC, 96:357-368, 1996.

[Yun et al., 2018] Bruno Yun, Srdjan Vesic, Madalina Croitoru, and Pierre Bisquert. Viewpoints using rankingbased argumentation semantics. In Computational Models of Argument - Proceedings of COMMA 2018, Warsaw, Poland, 12-14 September 2018, pages 381-392, 2018. 